\title{
Removal and Contraction for n-Dimensional Generalized Maps
}

\author{
Guillaume Damiand and Pascal Lienhardt \\ IRCOM-SIC, UMR-CNRS 6615 - bât. SP2MI, Bvd M. et P. Curie \\ BP 30179, 86962 Futuroscope Chasseneuil Cedex - France \\ \{damiand, lienhardt\}@sic.univ-poitiers.fr
}

\begin{abstract}
Removal and contraction are basic operations for several methods conceived in order to handle irregular image pyramids, for multi-level image analysis for instance. Such methods are often based upon graph-like representations which do not maintain all topological information, even for 2-dimensional images. We study the definitions of removal and contraction operations in the generalized maps framework. These combinatorial structures enable us to unambiguously represent the topology of a well-known class of subdivisions of $n$-dimensional (discrete) spaces. The results of this study make a basis for a further work about irregular pyramids of $n$-dimensional images.
\end{abstract}

Keywords. Removal, contraction, irregular pyramids, generalized maps.

\section{Introduction}

Many works deal with regular (cf. e.g. [1]) or irregular (cf. e.g. [2-4]) image pyramids for multi-level analysis and treatments (cf. also [5]). For irregular pyramids, it is necessary to handle a (topological) representation and basic operations, for instance dual graphs and removal and contraction operations for 2D images [6, 7]. Similar problems about multi-level representations arise also in geometric modeling (e.g. for CAD applications, architectural or geological modeling,...).

Our goal is to build a theoretical framework for the definition and handling of $n$-dimensional irregular pyramids: the $2 \mathrm{D}$ case has been widely studied; the importance of the 3D case is now well-known, and several works deal with 4D objects for which time corresponds to the fourth dimension (e.g. sequences of 3D images and 4D geometric modeling for animation). We think it is thus important to get coherent definitions of data structures and operations for any dimension.

So we study the definition of removal and contraction of $i$-dimensional cells within $n$-dimensional objects, in order to rigorously define the relations between two consecutive levels of a pyramid. This is a basic work which would enable us to conceive data structures (including their constraints of consistency), providing unique and unambiguous representations for pyramids of subdivided objects ${ }^{1}$.

\footnotetext{
${ }^{1}$ Informally, a subdivision of an $n$-dimensional space is a partition into $i$-dimensional cells (or $i$-cells), for $0 \leq i \leq n$.
} 
Our choice for the data structures for which we study the definitions of removal and contraction operations is mainly a consequence of the fact that unique and unambiguous representations are needed, which take into account multiadjacency. For instance, a main drawback of many graph-like representations is the fact that the whole topological information is often not maintained. More precisely, such representations are often ambiguous, even for 2-dimensional images, in particular when image regions are multi-adjacent. And multi-adjacency usually appears when constructing irregular pyramids.

The definition of subdivision representations is the subject of many works in the field of imagery, but also in the field of geometric modeling (cf. for instance [8-13]). Several representations extend the notion of $2 \mathrm{D}$ combinatorial map [14-16] for several classes of $n$-dimensional subdivisions [12,17]. Note also that several models based on combinatorial maps have been proposed for handling 2-dimensional $[18,19]$ and 3-dimensional segmented or multi-level images [20-24]. For instance, Brun and Kropatsch revisit works about graphs [25-28] in order to define 2-dimensional combinatorial map pyramids.

We choose to study the definitions of removal and contraction operations for $n$-dimensional generalized maps, since this notion enables us to unambiguously represent the topology of quasi-manifolds, which is a well-defined class of subdivisions [12]. Generalized maps are defined for any dimension, and their algebraic definition is simple; several kernels of geometric modeling softwares are based upon data structures derived from this notion. More precisely, generalized maps (resp. combinatorial maps) represent orientable or not orientable quasimanifolds, with or without boundaries (resp. orientable without boundaries). It is clear that most applications deal with orientable subdivisions without boundaries. Nevertheless, we mainly choose to deal with generalized maps rather than combinatorial maps or equivalent structures since we can provide simpler definitions of data structures and operations, and so more efficiency for the conception of softwares. Moreover, we known how to deduce combinatorial maps from generalized maps, so the results presented in this paper can be extended for combinatorial maps. Precise relations between generalized and combinatorial maps, and other classical data structures are presented in [29].

The main result of this paper consists in Def. 5 and Theorem 2 which shows that, for a given dimension $n$, we can simultaneously remove and contract cells of different dimensions under some simple conditions. This extends previous 2D and $3 \mathrm{D}$ results for the $n$-D case, and even for the more studied $2 \mathrm{D}$ and $3 \mathrm{D}$ cases (cf. $[25,20,21])$ this shows that more operations can be simultaneously applied (this is important for parallelization and for reducing the number of pyramid levels). More precisely, we define removal of one $i$-dimensional cell, and contraction by duality (cf. Sec. 3 and Sec. 4); according to the respective values of $i$ and $n$, a simple precondition is to be satisfied. Then we extend this definition in order to simultaneously remove and contract several $i$-cells for a given $i$. At last, we extend it for removing and contracting several cells of any dimensions (cf. Sec. 5): we show that it is possible if and only if cells are disjoined. 


\section{Generalized Maps Recall}

An $n$-dimensional generalized map is a set of abstract elements, called darts, and applications defined on these darts:

Definition 1 (Generalized map). Let $n \geq 0$. A n-dimensional generalized map (or n-G-map) is $G=\left(B, \alpha_{0}, \ldots, \alpha_{n}\right)$ where:

1. $B$ is a finite set of darts;

2. $\forall i, 0 \leq i \leq n, \alpha_{i}$ is an involution ${ }^{2}$ on $B$;

3. $\forall i, j, 0 \leq i<i+2 \leq j \leq n, \alpha_{i} \alpha_{j}$ is an involution.

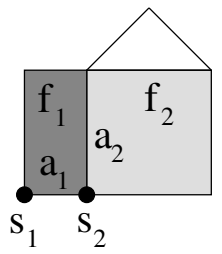

(a)

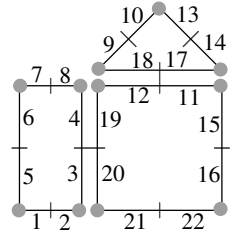

(b)

\begin{tabular}{|c|c|}
\hline & $\begin{array}{lllll}123 & 456\end{array}$ \\
\hline$\alpha_{0}$ & 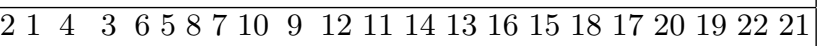 \\
\hline$\alpha_{1}$ & 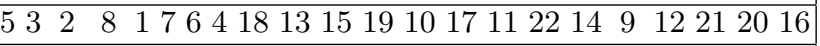 \\
\hline & 12 \\
\hline
\end{tabular}

Fig. 1. (a) A 2D subdivision. (b) The corresponding 2-G-map (involutions are given explicitly in the array). Darts are represented by numbered black segments. Two darts in relation by $\alpha_{0}$ share a little vertical segment (ex. darts 1 and 2). Two darts in relation by $\alpha_{1}$ share a same point (ex. darts 2 and 3). Two distinct darts in relation by $\alpha_{2}$ are parallel and close to each other (ex. darts 3 and 20); otherwise, the dart is its own image by $\alpha_{2}$ (ex. dart 2 ). Dart 1 corresponds to $\left(s_{1}, a_{1}, f_{1}\right)$, dart $2=1 \alpha_{0}$ corresponds to $\left(s_{2}, a_{1}, f_{1}\right), 3=2 \alpha_{1}$ corresponds to $\left(s_{2}, a_{2}, f_{1}\right)$, and $20=3 \alpha_{2}$ corresponds to $\left(s_{2}, a_{2}, f_{2}\right)$. The vertex incident to dart 2 is $\left\langle\alpha_{1}, \alpha_{2}\right\rangle(2)=\{2,3,20,21\}$, the edge incident to dart 3 is $\left\langle\alpha_{0}, \alpha_{2}>(3)=\{3,4,19,20\}\right.$, and the face incident to dart 9 is $\left\langle\alpha_{0}, \alpha_{1}\right\rangle$ $(9)=\{9,10,13,14,17,18\}$.

Let $\mathrm{G}$ be an $n$-G-map, and $\mathrm{S}$ be the corresponding subdivision. Intuitively, a dart of $\mathrm{G}$ corresponds to an $(n+1)$-tuple of cells $\left(c_{0}, \ldots, c_{n}\right)$, where $c_{i}$ is an $i$-dimensional cell that belongs to the boundary of $c_{i+1}$ (cf. [11] and Fig. 1). $\alpha_{i}$ associates darts corresponding with $\left(c_{0}, \ldots, c_{n}\right)$ and $\left(c_{0}^{\prime}, \ldots, c_{n}^{\prime}\right)$, where $c_{j}=c_{j}^{\prime}$ for $j \neq i$, and $c_{i} \neq c_{i}^{\prime}\left(\alpha_{i}\right.$ swaps the two $i$-cells that are incident to the same $(i-1)$ and $(i+1)$-cells). When two darts $b_{1}$ and $b_{2}$ are such that $b_{1} \alpha_{i}=b_{2}$ $(0 \leq i \leq n), b_{1}$ is $i$-sewn with $b_{2}$.

G-maps represent cells in an implicit way:

\footnotetext{
${ }^{2}$ An involution $f$ on $S$ is a one to one mapping from $S$ onto $S$ such that $f=f^{-1}$.
} 
Definition 2 (i-cell). Let $G$ be an $\mathrm{n}-G$-map, $b$ a dart and $i \in N=\{0, \ldots, n\}$. The $\mathrm{i}$-cell incident to $b$ is the orbit ${ }^{3}$

$$
<>_{N-\{i\}}(b)=<\alpha_{0}, \ldots, \alpha_{i-1}, \alpha_{i+1}, \ldots, \alpha_{n}>(b)
$$

Intuitively, an $i$-cell is the set of all darts which can be reached starting from $b$, by using any combination of all involutions except $\alpha_{i}$. The set of $i$-cells is a partition of the darts of the G-map, for each $i$ between 0 and $n$. Two cells are disjoined if their intersection is empty, i.e. when no dart is shared by the cells. More precisions about G-maps are provided in [12].

\section{Removal}

Intuitively and in a general way for an $n$-dimensional space, the removal of an $i$-cell consists in removing this cell and in merging its two incidents $(i+1)$-cells: so removal can be defined for $0 \ldots(n-1)$-cells.

\subsection{Dimension 1: 0-Removal}

For dimension 1 , only the 0 -removal exists, which consists in removing a vertex and in merging its two incident edges. Let $C=<\alpha_{1}>(b)$ be a vertex, let $C \alpha_{0}$ be the "neighbor" darts of $C$ for $\alpha_{0}$, i.e. $C \alpha_{0}=\left\{b^{\prime \prime} \mid \exists b^{\prime} \in C\right.$ such that $\left.b^{\prime} \alpha_{0}=b^{\prime \prime}\right\}$, and let $B^{S}=C \alpha_{0}-C$ be the "neighbor" darts of $C$ for $\alpha_{0}$ that do not belong to $C$ (see Fig. 2). The G-map resulting from the 0-removal of $C$ is obtained by redefining $\alpha_{0}$ for the darts of $B^{S}$ as follows: $\forall b^{\prime} \in B^{S}, b^{\prime} \alpha_{0}^{\prime}=b^{\prime}\left(\alpha_{0} \alpha_{1}\right)^{k} \alpha_{0}$, where $k$ is the smallest integer such that $b^{\prime}\left(\alpha_{0} \alpha_{1}\right)^{k} \alpha_{0} \in B^{S}$. Note that $\alpha_{1}$ is not modified by 0 -removal.

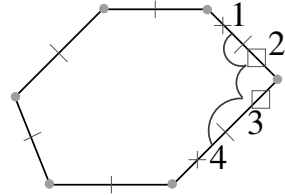

(a)

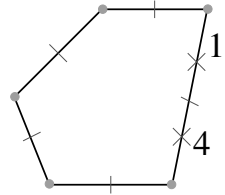

(b)

Fig. 2. 0-removal in 1D. (a) Initial 1-G-map. (b) Result. $C=<\alpha_{1}>(2)=\{2,3\}$ (darts marked with empty squares), $C \alpha_{0}=\{1,4\}=B^{S}$ (darts marked with crosses). 0-removal consists in setting $1 \alpha_{0}^{\prime}=1\left(\alpha_{0} \alpha_{1}\right) \alpha_{0}=4 \in B^{S}$ and $4 \alpha_{0}^{\prime}=4\left(\alpha_{0} \alpha_{1}\right) \alpha_{0}=1 \in$ $B^{S}$.

\footnotetext{
${ }^{3}$ Let $\left\{\Pi_{0}, \ldots, \Pi_{n}\right\}$ be a set of permutations on B. The orbit of an element $b$ relatively to this set of permutations is $<\Pi_{0}, \ldots, \Pi_{n}>(b)=\left\{\Phi(b), \Phi \in<\Pi_{0}, \ldots, \Pi_{n}>\right\}$, where $<\Pi_{0}, \ldots, \Pi_{n}>$ denotes the group of permutations generated by $\Pi_{0}, \ldots, \Pi_{n}$.
} 


\subsection{Dimension 2}

There are two different removal operations (0 and 1-removal) for dimension 2.

0-Removal It consists in removing a 0 -cell $C=<\alpha_{1}, \alpha_{2}>(b)$. Let $B^{S}=$ $C \alpha_{0}-C\left(C \alpha_{0}\right.$ is defined as above). This operation can be applied only if the following precondition is satisfied: $\forall b^{\prime} \in C, b^{\prime} \alpha_{1} \alpha_{2}=b^{\prime} \alpha_{2} \alpha_{1}$. This constraint corresponds, in the general case, to the fact that the degree of the vertex is equal to 2 ( 2 edges are incident to the vertex). If this constraint is not satisfied, we do not know how to join the cells incident to $C$, and it is then impossible to define the removal in a simple way. [30] proposes a generalization of this operation, but it is complex and cannot be used for an automatic process, in particular in automatic image processing.

The G-map resulting from 0-removal is obtained by redefining $\alpha_{0}$ for the darts of $B^{S}$ as follows: $\forall b^{\prime} \in B^{S}, b^{\prime} \alpha_{0}^{\prime}=b^{\prime}\left(\alpha_{0} \alpha_{1}\right)^{k} \alpha_{0}$ where $k$ is the smallest integer such that $b^{\prime}\left(\alpha_{0} \alpha_{1}\right)^{k} \alpha_{0} \in B^{S}$. Note that this redefinition of $\alpha_{0}$ is the same as for dimension 1 but concerns different darts, since it is a 0-cell within a 2D object: cf. Fig. 3 (intuitively, in the general case, this operation consists in applying twice the 0-removal defined for dimension 1).

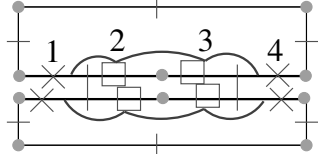

(a)

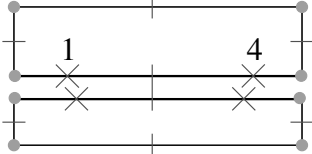

(b)

Fig. 3. 0-removal in 2D. (a) Initial 2-G-map. (b) Result. $C=<\alpha_{1}, \alpha_{2}>$ (2) (darts marked with empty squares), $C \alpha_{0}=B^{S}$ (darts marked with crosses). For instance, $1 \alpha_{0}^{\prime}=1\left(\alpha_{0} \alpha_{1}\right) \alpha_{0}=4 \in B^{S}$

1-Removal It consists in removing a 1-cell $C=<\alpha_{0}, \alpha_{2}>(b)$. This can be achieved without any precondition. Let $B^{S}=C \alpha_{1}-C$. The resulting G-map is obtained by redefining $\alpha_{1}$ for the darts of $B^{S}$ as follows: $\forall b^{\prime} \in B^{S}, b^{\prime} \alpha_{1}^{\prime}=$ $b^{\prime}\left(\alpha_{1} \alpha_{2}\right)^{k} \alpha_{1}$, where $k$ is the smallest integer such that $b^{\prime}\left(\alpha_{1} \alpha_{2}\right)^{k} \alpha_{1} \in B^{S}$. Examples of 1-removal are presented in Fig. 4 , and 5. For this last example, $k=2$ since the removed edge is incident twice to the same vertex.

\subsection{Dimension $\mathbf{n}$}

The general definition of $i$-cell removal for an $n$-dimensional G-map is an obvious extension of the previous cases. Let $C$ be an $i$-cell to remove; when $i<n-1$ 


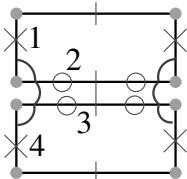

(a)

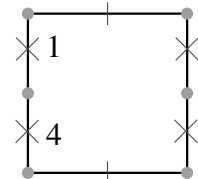

(b)

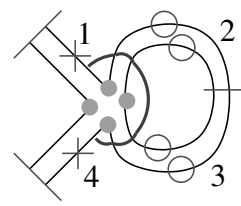

(a)

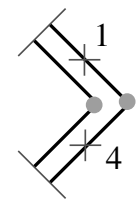

(b)
Fig. 4. 1-removal in $2 \mathrm{D}$ in the general case. Darts of the edge to remove are marked with circles. (a) Initial 2-G-map. (b) Result.
Fig. 5. 1-removal in $2 \mathrm{D}$ of a loop. (a) Initial 2-G-map. (b) Result. For instance, $1 \alpha_{1}^{\prime}=$ $1\left(\alpha_{1} \alpha_{2}\right)\left(\alpha_{1} \alpha_{2}\right) \alpha_{1}=4 \in B^{S}$ (since $1\left(\alpha_{1} \alpha_{2}\right) \alpha_{1} \notin$ $B^{S}$, this dart belongs either to $C$ and to $\left.C \alpha_{1}\right)$.

the operation can be applied only when, informally ${ }^{4}$ the degree of $C$ is equal to 2 (a vertex incident to exactly two edges or an edge incident to two faces or a face incident to two volumes...). The $i$-removal consists then in redefining $\alpha_{i}$ for the darts of $B^{S}=C \alpha_{i}-C$ in the following way: $b^{\prime} \alpha_{i}^{\prime}=b^{\prime}\left(\alpha_{i} \alpha_{i+1}\right)^{k} \alpha_{i}$, where $k$ is the smallest integer such that $b^{\prime}\left(\alpha_{i} \alpha_{i+1}\right)^{k} \alpha_{i} \in B^{S}$. We obtain so the general definition of the $i$-removal operation:

Definition 3 (i-cell removal). Let $G=\left(B, \alpha_{0}, \ldots, \alpha_{n}\right)$ be an $\mathrm{n}-G$-map, $i \in$ $\{0, \ldots, n-1\}$ and $C=<>_{N-\{i\}}(b)$ be an i-cell, such that: $\forall b^{\prime} \in C, b^{\prime} \alpha_{i+1} \alpha_{i+2}=$ $b^{\prime} \alpha_{i+2} \alpha_{i+1}$. Let $B^{S}=C \alpha_{i}-C$, the set of darts i-sewn to $C$ that do not belong to $C$. The $\mathrm{n}$-G-map resulting from the removal of this $\mathrm{i}$-cell is $G^{\prime}=\left(B^{\prime}, \alpha_{0}^{\prime}, \ldots, \alpha_{n}^{\prime}\right)$ defined by:

$-B^{\prime}=B-C$;

$-\forall j \in\{0, \ldots, n\}-\{i\}, \alpha_{j}^{\prime}=\left.\alpha_{j}\right|_{B^{\prime}} ;^{5}$

$-\forall b^{\prime} \in B^{\prime}-B^{S}, b^{\prime} \alpha_{i}^{\prime}=b^{\prime} \alpha_{i}$

$-\forall b^{\prime} \in B^{S}, b^{\prime} \alpha_{i}^{\prime}=b^{\prime}\left(\alpha_{i} \alpha_{i+1}\right)^{k} \alpha_{i}$, where $k$ is the smallest integer such that $b^{\prime}\left(\alpha_{i} \alpha_{i+1}\right)^{k} \alpha_{i} \in B^{S}$.

Theorem 1. $G^{\prime}$ is an n-G-map.

Proof. It is easy to check that $G^{\prime}$ satisfies conditions 2 and 3 of Def. 1. cf. [31].

Note that $G^{\prime}$ can contain only one $n$-cell, and may even be empty if $G$ contains only one $i$-cell.

\section{Contraction}

Informally, $i$-contraction consists in contracting an $i$-cell into an $(i-1)$-cell. Contraction is the dual of the removal operation. Informally, the dual of a subdivision is a subdivision of the same space, in which an $(n-i)$-cell is associated with

\footnotetext{
4 The formal precondition is: $\forall b^{\prime} \in C, b^{\prime} \alpha_{i+1} \alpha_{i+2}=b^{\prime} \alpha_{i+2} \alpha_{i+1}$. Note that if $i=n-1$ this condition doesn't apply and we can always remove any $(n-1)$-dimensional cell. ${ }^{5} \alpha_{j}^{\prime}$ is equal to $\alpha_{j}$ restricted to $B^{\prime}$, i.e. $\forall b \in B^{\prime}, b \alpha_{i}^{\prime}=b \alpha_{i}$
} 
each initial $i$-cell, and incidence relations are kept. A nice property of G-maps is the fact that the dual G-map of $G=\left(B, \alpha_{0}, \ldots, \alpha_{n}\right)$ is $G^{\prime}=\left(B, \alpha_{n}, \ldots, \alpha_{0}\right)$ : we just need to reverse the involution order.

We can thus easily deduce the definition of $i$-contraction from the general definition of $i$-removal. We just have to replace '+' by '-' for indices of involutions for preconditions and operations, i.e. $\alpha_{i+1} \alpha_{i+2} \rightarrow \alpha_{i-1} \alpha_{i-2}$ and $\alpha_{i} \alpha_{i+1} \rightarrow \alpha_{i} \alpha_{i-1}$. (see two examples of contraction in Fig. 6 and 7 ).

Definition 4 (i-cell contraction). Let $G=\left(B, \alpha_{0}, \ldots, \alpha_{n}\right)$ be an $\mathrm{n}-G$-map, $i \in\{1, \ldots, n\}$ and $C=<>_{N-\{i\}}(b)$ be an i-cell, such that ${ }^{6}: \forall b^{\prime} \in C, b^{\prime} \alpha_{i-1} \alpha_{i-2}=$ $b^{\prime} \alpha_{i-2} \alpha_{i-1}$. Let $B^{S}=C \alpha_{i}-C$, the set of darts i-sewn to $C$ that do not belong to $C$. The n-G-map resulting from the contraction of this i-cell is $G^{\prime}=$ $\left(B^{\prime}, \alpha_{0}^{\prime}, \ldots, \alpha_{n}^{\prime}\right)$ defined by:

$-B^{\prime}=B-C$;

$-\forall j \in\{0, \ldots, n\}-\{i\}, \alpha_{j}^{\prime}=\left.\alpha_{j}\right|_{B^{\prime}}$;

$-\forall b^{\prime} \in B^{\prime}-B^{S}, b^{\prime} \alpha_{i}^{\prime}=b^{\prime} \alpha_{i}$;

$-\forall b^{\prime} \in B^{S}, b^{\prime} \alpha_{i}^{\prime}=b^{\prime}\left(\alpha_{i} \alpha_{i-1}\right)^{k} \alpha_{i}$, where $k$ is the smallest integer such that $b^{\prime}\left(\alpha_{i} \alpha_{i-1}\right)^{k} \alpha_{i} \in B^{S}$.

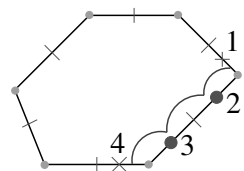

(a)

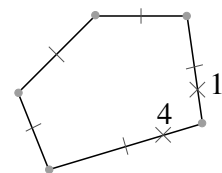

(b)

Fig. 6. 1-contraction in 1D. Darts of the edge to contract are marked with black disks. (a) Initial 2-G-map. (b) Result.

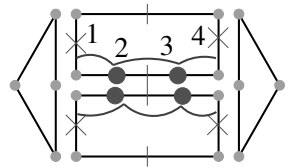

(a)

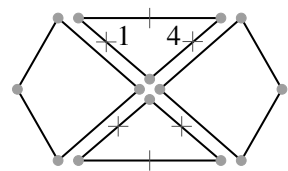

(b)

Fig. 7. 1-contraction in 2D. (a) Initial 2-Gmap. (b) Result.

\section{Generalisations}

Previous definitions enable us to remove or to contract a single cell. For some applications, it could be more efficient to simultaneously apply several operations. Concretely, let $G$ be an $n$-G-map. Assume that each dart belonging to a removed or contracted cell is marked with the dimension and type of the corresponding operation. Operations can be simultaneously applied if and only if:

- the cells are disjoined (involving that a dart has at most a unique mark and thus that there is no ambiguity when redefining $\alpha_{i}$ for each $i, 0 \leq i \leq n$ );

- the preconditions of the corresponding operations are satisfied.

\footnotetext{
${ }^{6}$ Note that this condition doesn't apply for $i=1$, so we can always contract any edge.
} 
Indeed, when a precondition is satisfied before a set of operations, this precondition is still satisfied after each step of the operation (the converse is obviously false), because cells are disjoined. This allows us to apply simultaneously or successively a set of operations and to obtain the same result.

We now present this generalization in several steps. First, we show that it is possible to simultaneously perform removals (resp. contractions) of several $i$-cells for a given $i(0 \leq i \leq n)$.

Generalisation 1 We can easily prove that the previous definition of removal (resp. contraction) stands for the removal (resp. contraction) of a set of cells of same dimension $i$. The (possible) precondition of the initial operation has to be satisfied for each cell (cf. Fig. 8(a)).

Moreover, removing (resp. contracting) simultaneously several $i$-cells or applying successively and in any order the initial operation for any removed cell, produce the same result. The main idea of the proof is: each $\alpha_{i}$ redefinition consists in: $\forall b \in B^{S i}, b \alpha_{i}^{\prime}=b\left(\alpha_{i} \alpha_{i+1}\right)^{k} \alpha_{i}$. The darts of this path can be partitioned depending on the removed cells they belong to, i.e. $b \alpha_{i}^{\prime}=b\left(\alpha_{i} \alpha_{i+1}\right)^{k_{1}}\left(\alpha_{i} \alpha_{i+1}\right)^{k_{2}}$ $\ldots\left(\alpha_{i} \alpha_{i+1}\right)^{k_{p}} \alpha_{i}$. Each subpath corresponds to a single removal and so order is not important, and each removal do not depend on other operations.

We retrieve here the notion of connecting walk of Brun and Kropatsch [26]. Darts of $B^{S} i$ are surviving darts and $\alpha_{i}^{\prime}$ put in relation two darts of $B^{S} i$ by traversing some non-surviving darts (darts traversed by $\left.\left(\alpha_{i} \alpha_{i+1}\right)^{k}\right)$.

Generalisation 2 The previous generalization can be directly extended for simultaneously removing and contracting cells of same dimension $i$. A cell is either removed or contracted, but not both at the same time. The (possible) precondition of the corresponding initial operation has to be satisfied for each cell (cf. Fig. 8(b)).

More precisely, let $C S^{i}$ (resp. $C C^{i}$ ) be a set of $i$-cells to remove (resp. contract), such that $C S^{i} \cap C C^{i}=\emptyset$ and such that the (possible) precondition of $i$-removal (resp. $i$-contraction) operation is satisfied for each cell of $C S^{i}$ (resp. $\left.C C^{i}\right)$. Let $B^{S i}=\left(C C^{i} \cup C S^{i}\right) \alpha_{i}-\left(C C^{i} \cup C S^{i}\right)$. As before, $\alpha_{i}$ is redefined for these darts: $\forall b \in B^{S i}, b \alpha_{i}^{\prime}=b^{\prime}=b\left(\alpha_{i} \alpha_{k_{1}}\right) \ldots\left(\alpha_{i} \alpha_{k_{p}}\right) \alpha_{i}$ where $p$ is the smallest integer such that $b^{\prime} \in B^{S i}$ and $\forall j, 1 \leq j<p$, if $b_{c}=b\left(\alpha_{i} \alpha_{k_{1}}\right) \ldots\left(\alpha_{i} \alpha_{k_{j-1}}\right) \alpha_{i} \in C S^{i}$ then $k_{j}=i+1$ else $\left(b_{c} \in C C^{i}\right) k_{j}=i-1$.

Generalisation 3 The previous generalization can be directly extended for the removal and/or contraction of a set of disjoined cells of any dimension. The (possible) precondition of the corresponding initial operation has to be satisfied for each cell.

This last generalization is possible because the set of cells are disjoined. If we consider two disjoined cells, there is two possibilities: the two cells have the same dimension and they can be adjacent or not; the two cells have different dimensions and they can not be incident. The first case is covered by the previous 


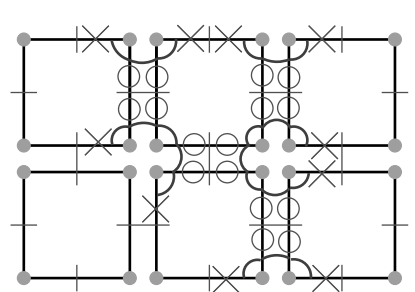

(a)

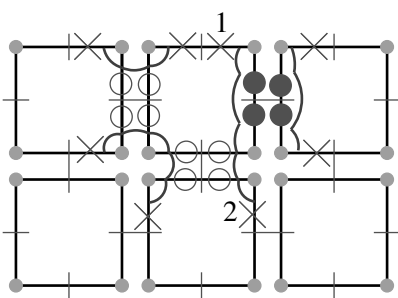

(b)

Fig. 8. (a) Simultaneous 1-removals in 2D. (b) Simultaneous 1-removals (dart marked with circles) and 1-contractions (dart marked with black disks) in 2D. For instance, $1 \alpha_{1}^{\prime}=1\left(\alpha_{1} \alpha_{0}\right)\left(\alpha_{1} \alpha_{2}\right) \alpha_{1}=2$ since the edge incident to $1 \alpha_{1}$ is contracted and the edge incident to $1\left(\alpha_{1} \alpha_{0}\right) \alpha_{1}$ is removed.

generalizations. The second one is easy to consider since the cells are not incident: so some surviving darts exist between the two cells, and $\alpha_{i}$ is redefined only for these darts. The other cells are not modified and so other preconditions are still valid when applying any subset of these operations.

The following definition covers all the previous operations. As for the previous generalizations, $\alpha_{i}$ is redefined only for the darts of $B^{S i}$, but this redefinition is now done for any $i, 0 \leq i \leq n$.

Definition 5 (Simultaneous removal and contraction of cells of any dimension).

Let $G=\left(B, \alpha_{0}, \ldots, \alpha_{n}\right)$ be an $\mathrm{n}-G$-map, $C S^{0}, \ldots, C S^{n-1}$ be sets of 0 -cells, $\ldots$, $(n-1)$-cells to be removed and $C C^{1}, \ldots, C C^{n}$ be sets of 1 -cells, $\ldots, \mathrm{n}$-cells to be contracted. Let $C S=\cup_{i=0}^{n-1} C S^{i}$ and $C C=\cup_{i=1}^{n} C C^{i}$. Two preconditions have to be satisfied: cells are disjoined (i.e. $\forall C, C^{\prime} \in C C \cup C S, C \cap C^{\prime}=\emptyset$ ), and "the degree of each cell is equal to 2", i.e.:

- $\forall i, 0 \leq i \leq n-2, \forall b \in C S^{i}, b \alpha_{i+1} \alpha_{i+2}=b \alpha_{i+2} \alpha_{i+1}$

- $\forall i, 2 \leq i \leq n, \forall b \in C C^{i}, b \alpha_{i-1} \alpha_{i-2}=b \alpha_{i-2} \alpha_{i-1}$

Let $B^{S i}=\left(C S^{i} \cup C C^{i}\right) \alpha_{i}-\left(C S^{i} \cup C C^{i}\right) \forall i, 0 \leq i \leq n$. The resulting n-G-map is $G^{\prime}=\left(B^{\prime}, \alpha_{0}^{\prime}, \ldots, \alpha_{n}^{\prime}\right)$ defined by:

- $B^{\prime}=B-(C C \cup C S)$;

- $\forall i, 0 \leq i \leq n, \forall b \in B^{\prime}-B^{S i}, b \alpha_{i}^{\prime}=b \alpha_{i}$;

- $\forall i, 0 \leq i \leq n, \forall b \in B^{S i}, b \alpha_{i}^{\prime}=b^{\prime}=b\left(\alpha_{i} \alpha_{k_{1}}\right) \ldots\left(\alpha_{i} \alpha_{k_{p}}\right) \alpha_{i}$, where $p$ is the smallest integer such that $b^{\prime} \in B^{S i}$, and $\forall j, 1 \leq j<p$, if $b_{c}=$ $b\left(\alpha_{i} \alpha_{k_{1}}\right) \ldots\left(\alpha_{i} \alpha_{k_{j-1}}\right) \alpha_{i} \in C S^{i}$ then $k_{j}=i+1$ else $\left(b_{c} \in C C^{i}\right) k_{j}=i-1$.

Theorem 2. The general removal and contraction operation produces an n-Gmap.

Proof. cf. [31].

An example of this last generalization is given in Fig. 9, for which all possible removal and contraction operations are simultaneously applied. We can check 


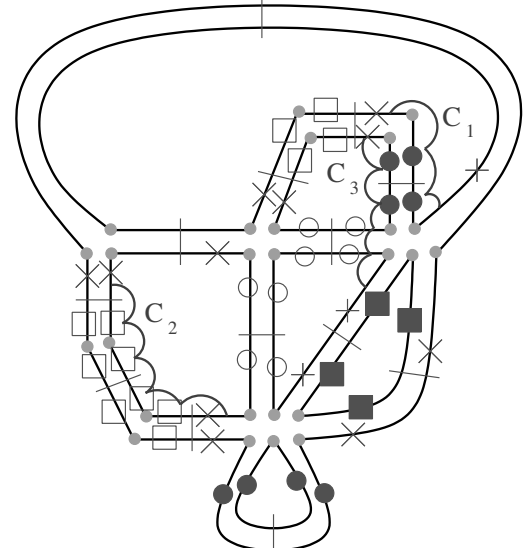

(a)

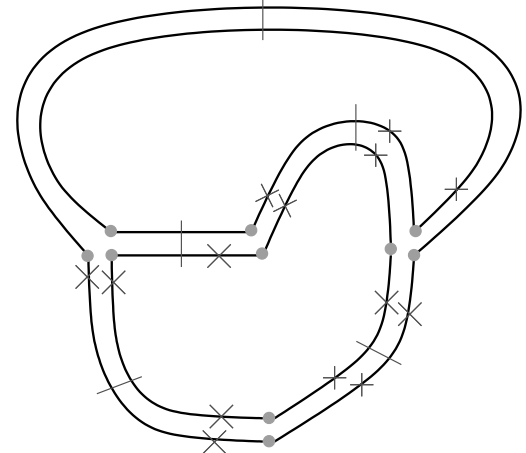

(b)

Fig. 9. An example in $2 \mathrm{D}$ of simultaneous removal and contraction of cells of different dimensions. (a) 2-G-map before operations. (b) The resulting 2-G-map. Darts belonging to a removed 1-cell (resp. removed 0-cell, contracted 1-cell, contracted 2-cell) are marked with a circle (resp. an empty square, a black disk, a filled square). Darts marked with crosses belong to $\cup B^{S i}$. Three connecting walks are represented: $C_{1}$ which traverses a contracted edge, $C_{2}$ which traverses two contracted vertices and $C_{3}$ which traverses one contracted edge then one removed edge.

that we get the same result when cells are successively removed or contracted by the initial operation in any order. We retrieve here the notion of connecting dart sequence of Brun and Kropatsch [32].

\section{Conclusion and Perspectives}

In this paper, we have defined removal and contraction operations, which can be applied to any cells of any G-maps, whatever their respective dimensions. Moreover, we have studied how to perform simultaneously different operations. These definitions are homogeneous for any dimension. Since combinatorial maps [20, 25 ] can be easily deduced from orientable generalized maps [12], these operations can also be defined on combinatorial maps.

We intend to revisit the works of Brun and Kropatsch for handling irregular pyramids of $n$-dimensional generalized maps. Properties of removal and contraction operations would enable us to establish relations between two contiguous levels within a pyramid, and thus between any levels. Efficient data structures could be deduced taking these relations into account.

Theorem 2 means that the result of removal and contraction operations is a valid object. However, topological properties (as connectivity for instance) 
could be not preserved when applying these operations. Since preserving topological properties is an essential issue, in particular for image pyramids, we are studying the evolution of some topological characteristics, in order to control the construction of coherent pyramids. Some results are presented in [24] in the particular framework of 2D and 3D images representation; they have to be generalized in upper dimension and for the general case: since we exactly know the mathematical objects associated with G-maps, it is possible to apply well-known results of combinatorial topology in order to control the evolution of topological properties.

In order to conceive efficient algorithms, another interesting perspective is the parallelization of the application of a set of operations. We think that, in the general case, checking preconditions could be distributed on cells: it is then possible to simultaneously compute sets $C \alpha_{i}-C$; the application of the operations could be distributed on the surviving darts (but this has to be more deeply studied). It is also necessary to study particular cases, for instance when removed or contracted cells satisfy some particular properties (a well known example consists in removing a tree of edges).

\section{References}

1. Burt, P., Hong, T.H., Rosenfeld, A.: Segmentation and estimation of image region properties through cooperative hierarchical computation. IEEE Transactions on Systems, Man and Cybernetics 11(12) (december 1981) 802-809

2. Meer, P.: Stochastic image pyramids. Computer Vision, Graphics and Image Processing 45 (1989) 269-294

3. Montanvert, A., Meer, P., Rosenfeld, A.: Hierarchical image analysis using irregular tesselations. IEEE Transactions on Pattern Analysis and Machine Intelligence 13(4) (april 1991) 307-316

4. Jolion, J., Montanvert, A.: The adaptive pyramid : a framework for $2 \mathrm{~d}$ image analysis. Computer Vision, Graphics and Image Processing 55(3) (may 1992) 339-348

5. Kropatsch, W.: Abstraction pyramids on discrete representations. In: Discrete Geometry for Computer Imagery. Number 2301 in LNCS, Bordeaux, France (april 2002) 1-21

6. Kropatsch, W.: Building irregular pyramids by dual graph contraction. Technical report PRIP-TR-35, Dept. for Pattern Recognition and Image Processing, Institute for Automation, Technical Univerity of Vienna, Austria (july 1994)

7. Kropatsch, W.: Building irregular pyramids by dual-graph contraction. Vision, Image and Signal Processing 142(6) (december 1995) 366-374

8. Baumgart, B.: A polyhedron representation for computer vision. In: AFIPS nat. conf. Number 44 (1975) 589-596

9. Guibas, L., Stolfi, J.: Primitives for the manipulation of general subdivisions and the computation of voronoï diagrams. ACM Transactions on Graphics 4(2) (april 1985) $74-123$

10. Dobkin, D., Laszlo, M.: Primitives for the manipulation of three-dimensional subdivisions. Algorithmica 4(1) (1989) 3-32

11. Brisson, E.: Representing geometric structures in d dimensions: topology and order. Discrete Comput. Geom. 9(1) (1993) 387-426 
12. Lienhardt, P.: N-dimensional generalized combinatorial maps and cellular quasimanifolds. International Journal of Computational Geometry and Applications 4(3) (1994) 275-324

13. de Floriani, L., Mesmoudi, M., Morando, F., Puppo, E.: Non-manifold decomposition in arbitrary dimensions. In: Discrete Geometry for Computer Imagery. Number 2301 in LNCS, Bordeaux, France (april 2002) 69-80

14. Edmonds, J.: A combinatorial representation for polyhedral surfaces. Notices of the American Mathematical Society 7 (1960)

15. Jacques, A.: Constellations et graphes topologiques. In: Combinatorial Theory and Applications. Volume 2. (1970) 657-673

16. Cori, R.: Un code pour les graphes planaires et ses applications. PhD thesis, Universit de Paris VII (1973)

17. Elter, H., Lienhardt, P.: Cellular complexes as structured semi-simplicial sets. International Journal of Shape Modeling 1(2) (1994) 191-217

18. Brun, L.: Segmentation d'images couleur à base topologique. PhD thesis, Université de Bordeaux I (december 1996)

19. Fiorio, C.: Approche interpixel en analyse d'images : une topologie et des algorithmes de segmentation. PhD thesis, Université Montpellier II (24 novembre 1995)

20. Braquelaire, J., Desbarats, P., Domenger, J., Wüthrich, C.: A topological structuring for aggregates of $3 \mathrm{~d}$ discrete objects. In: Workshop on Graph based representations, Austria, IAPR-TC15 (may 1999) 193-202

21. Bertrand, Y., Damiand, G., Fiorio, C.: Topological encoding of 3d segmented images. In: Discrete Geometry for Computer Imagery. Number 1953 in LNCS, Uppsala, Sweden (december 2000) 311-324

22. Bertrand, Y., Damiand, G., Fiorio, C.: Topological map: minimal encoding of 3d segmented images. In: Workshop on Graph based representations, Ischia, Italy, IAPR-TC15 (may 2001) 64-73

23. Braquelaire, J., Desbarats, P., Domenger, J.: 3d split and merge with 3-maps. In: Workshop on Graph based representations, Ischia, Italy, IAPR-TC15 (may 2001) $32-43$

24. Damiand, G.: Définition et étude d'un modèle topologique minimal de représentation d'images $2 \mathrm{~d}$ et $3 \mathrm{~d}$. PhD thesis, Université de Montpellier II (december 2001)

25. Brun, L., Kropatsch, W.: Dual contraction of combinatorial maps. In: Workshop on Graph based representations, Austria, IAPR-TC15 (may 1999) 145-154

26. Brun, L., Kropatsch, W.: Pyramids with combinatorial maps. Technical report 57, Institute of Computer Aided Automation, Vienna University of Technology, Austria (december 1999) URL: http://www.prip.tuwien.ac.at/.

27. Brun, L., Kropatsch, W.: The construction of pyramids with combinatorial maps. Technical report 63, Institute of Computer Aided Automation, Vienna University of Technology, Austria (june 2000) URL: http://www.prip.tuwien.ac.at/.

28. Brun, L., Kropatsch, W.: Contraction kernels and combinatorial maps. In: Workshop on Graph based representations, Ischia, Italy, IAPR-TC15 (may 2001) 12-21

29. Lienhardt, P.: Topological models for boundary representation: a comparison with n-dimensional generalized maps. Commputer Aided Design 23(1) (1991) 59-82

30. Elter, H.: Etude de structures combinatoires pour la représentation de complexes cellulaires. PhD thesis, Université Louis-Pasteur, Strasbourg (september 1994)

31. Damiand, G., Lienhardt, P.: Removal and contraction for n-dimensional generalized maps. Technical Report 2003-01, Laboratoire IRCOM-SIC (january 2003) URL: http://damiands.free.fr/. 
32. Brun, L., Kropatsch, W.: Receptive fields within the combinatorial pyramid framework. In: Discrete Geometry for Computer Imagery. Number 2301 in LNCS, Bordeaux, France (april 2002) 92-101 